\title{
Dynamics of Spreading of Chainlike Molecules with Asymmetric Surface Interactions
}

\author{
M. Haataja, ${ }^{1,2}$ J. A. Nieminen, ${ }^{2}$ and T. Ala-Nissila ${ }^{1-3}$ \\ ${ }^{1}$ Research Institute for Theoretical Physics, \\ P.O. Box 9 (Siltavuorenpenger 20 C), FIN-00014 University of Helsinki, Finland \\ ${ }^{2}$ Laboratory of Physics, Tampere University of Technology, \\ P.O. Box 692, FIN-33101 Tampere, Finland \\ ${ }^{3}$ Department of Physics, Brown University, Providence, Rhode Island 02912, U.S.A.
}

(January 25, 1996)

In this work we study the spreading dynamics of tiny liquid droplets on solid surfaces in the case where the ends of the molecules feel different interactions with respect to the surface. We consider a simple model of dimers and short chainlike molecules that cannot form chemical bonds with the surface. We use constant temperature Molecular Dynamics techniques to examine in detail the microscopic structure of the time dependent precursor film. We find that in some cases it can exhibit a high degree of local order that can persist even for flexible chains. Our model also reproduces the experimentally observed early and late-time spreading regimes where the radius of the film grows $\propto t^{0.5}$. The ratios of the associated transport coefficients are in good overall agreement with experiments. Our density profiles are also in good agreement with measurements on the spreading of molecules on hydrophobic surfaces.

68.10.Gw, 61.20.Ja, 05.70.Ln

\section{INTRODUCTION}

Since the pioneering spreading experiments of microscopic liquid droplets on surfaces by Heslot et al. [1], there has been increasing interest in phenomena occuring at microscopic lengthscales during spreading. The experiments of Refs. [1] reveal that both the molecular structure of the liquid and the type of substrate used influence density profiles of the droplets. For example, thickness profiles of tetrakis (2-ethylexoxy)-silane and polydimethylsiloxane (PDMS) droplets on a silicon wafer exhibit strikingly different shapes under spreading [i]. Tetrakis exhibits clearly observable dynamical layering, while the spreading of PDMS proceeds by a quickly evolving precursor layer of one molecular thickness. Furthermore, the experiments of Valignat et al. [5] and Cazabat et al. [6] address the important role of surface grafting, and asymmetrical surface interactions in particular, on the spreading dynamics. For example, in Ref. [6] the density profiles for trisiloxane polyoxyethylene droplets spreading on a hydrophilic surface resemble a "sand pile", whereas the same liquid forms a very compact bilayer on a hydrophobic surface.

Despite drastic differences in the density profiles, an interesting feature in the experiments of Refs. [1 3 , 5. 5 is that the time dependence of the radius of the precursor film $r(t)$ follows "diffusive" behavior $r(t) \sim t^{0.5}$ for all times measured. Moreover, the experiments of Valignat et al. [5] report two distinct "diffusive" regimes comprising a rapid early-time region followed by a considerably slower late-time one. Typical estimates for the ratio between the corresponding early-time and late-time transport coefficients are of the order of 100 . The emergence of the early-time regime can most simply be explained by assuming that the flux onto the surface is constant, i.e. $d N_{p}(t) / d t=$ const., where $N_{p}(t)$ is the number of molecules in the effectively 2D precursor film [7]. From this it follows that $r(t) \sim \sqrt{N_{p}(t)} \sim t^{0.5}$. The late-time behavior is due to crossover towards $2 \mathrm{D}$ diffusion that takes over in the submonolayer regime [4, 8].

Motivated by these experimental discoveries a number of theoretical models have been proposed. However, progress has been rather moderate. Analytic theories to date deal with dynamical layering only [9.10]. Abraham et al. [9] considered a solid-on-solid (SOS) model of a layered droplet in rectangular geometry. The width of the precursor film was found to evolve linearily in time, implying that the effective flux of the liquid into the precursor film is indeed constant in time. De Gennes and Cazabat [10] considered a model in which the layers have already formed. This model gives simple relations for the time-dependence of the radii of the layers. In particular, for a completely layered droplet, the precursor film develops approximately "diffusively" in time [11]. However, both models treat the liquid as structureless and therefore cannot be applied to studying the effects of molecular structure or details of interactions on the spreading dynamics.

For both coarse-grained and more microscopic models, a number of computer simulations have been performed 1219 to study the dynamics of spreading. In particular, using Molecular Dynamics (MD) simulations it was concluded in Ref. [16] that both the chain-like nature of the molecules and the chain-surface interactions can significantly influence the structure of the precursor layer. Using a cylindrical droplet geometry, Refs. [14, 16] reported an "almost linear" early time regime for the width $w(t)$ of their precursor film (again indicating a constant flux), followed by a late-time diffusive region. Most recently, the $t^{0.5}$ behavior was observed in another MD simulation [19]. Qualitatively, the two time regimes have been seen 
in Monte Carlo simulations, too 15, 17.

In the present work, our aim is to employ MD simulation techniques to study a particularly interesting aspect of droplet spreading, namely the case where the fluid molecules can feel asymmetric interactions with respect to the surface. We will concentrate mostly on the microscopic structure of the precursor film, its time dependence and the quantitative evaluation of the associated transport coefficients. Our work is motivated by recent experiments on such systems [5.,6], as well as the practical importance of the molecular structure of thin layers. In particular, a common way of controlling the surface energetics of a solid substrate is to use grafted molecules that adsorb on it, sometimes forming chemical bonds and brushed layers [20. Such surfaces have important applications in e.g. coating and lubrication. Another interesting class of systems comprises amphiphilic molecules such as detergents where a strong asymmetry of interactions causes layered structures to form [21]. Spreading dynamics of such molecules is then of particular interest in trying to understand how these layers form, and how well ordered they are.

The outline of this paper is as follows. First, we introduce the model in detail. Then we present results for the spreading dynamics of molecules consisting of two, four, and eight units with an asymmetry of interaction with respect to the underlying surface. Our results reveal that in some cases the emerging precursor layer may exhibit a high degree of local order that persists even for the longer chains. This is reflected in the corresponding density profiles, some of which bear a close resemblance to the experimental ones [6]. We also examine the time dependence of the radius of the precursor layer, and find the two different "diffusive" regimes in agreement with previous studies [14,16] and experiments [5]. We furthermore evaluate the corresponding transport coefficients and find that their ratio is in good qualitative agreement with the experiments. Finally, we briefly discuss the influence of the choice of thermostat in our model. A brief account for the results for dimers has been previously given in Ref. [22].

\section{MODEL}

\section{A. Interactions}

Our MD model is analogous to the one introduced in Refs. [14,16]. The $n-$ mer molecules consist of $n$ LennardJones (LJ) particles. Within the chain, the LJ interaction between them is purely repulsive, i.e. of the form $V_{L J}^{\text {intra }}(r)=4 \epsilon_{f}\left(\sigma_{f} / r\right)^{12}$ to prevent spatial overlap. The potential parameters are $\sigma_{f}=2.3 \AA$ for the width and $\epsilon_{f}=0.1703 \mathrm{eV}$ for the depth, respectively. Additionally, the particles are interconnected by a very rigid but orientationally isotropic harmonic oscillator pair potential $V_{c}=\frac{1}{2} k\left(r-r_{0}\right)^{2}$, where $k=10000 \epsilon_{f} / \sigma_{f}^{2}$ and $r_{0}=2^{1 / 6} \sigma_{f}$ so that the chains do not easily stretch. There is also an angle dependent potential $V_{\theta}=\epsilon_{\theta}(\cos \theta+1)$, for $n>2$. For our studies we examine two cases, namely $\epsilon_{\theta}=10 \epsilon_{f}$ (rather stiff chains) and $\epsilon_{\theta}=0$ (completely flexible chains). There is no torsion dependent potential within a chain since we consider linear chains.

Interchain interactions are modeled by the following pairwise LJ interaction between $n$ LJ monomers:

$$
V_{L J}(r)=4 \epsilon_{f}\left[\left(\frac{\sigma_{f}}{r}\right)^{12}-\left(\frac{\sigma_{f}}{r}\right)^{6}\right] .
$$

The substrate on the other hand is modeled by a flat continuum LJ material; it is thought to be homogeneous and its unit volume $\Omega=1$. The total substrate interaction is obtained by integrating the LJ potential over the half space $z \leq 0$ with the result

$$
V_{i}(z)=-\frac{A}{z^{3}}+\frac{B}{z^{9}},
$$

where

$$
A=(2 \pi / 3) \rho_{s} \epsilon_{i} \sigma_{i}^{6} \text { and } B=(4 \pi / 45) \rho_{s} \epsilon_{i} \sigma_{i}^{12},
$$

and where $\rho_{s}$ denotes the number density of particles comprising the substrate. Different chain-surface interaction parameters employed in this study are presented in Table I and shown in Fig. 1.

The asymmetrical nature of the chain-surface interactions comes about through the choice of different set of surface interaction parameters for the grafted end as compared to the other monomer units along the chain. The "grafted" end interaction is set to $V_{\mathrm{I}}$ in every case studied in this work, whereas for dimers we employ $V_{\text {II }}$ (ordinary case) and $V_{\mathrm{III}}$ (shifted case), for tetramers $V_{\mathrm{IV}}$ (ordinary case) and $V_{\mathrm{V}}$ (shifted case), and for octamers $V_{\text {IV }}$. These potentials have been constructed in such a way that in the ordinary case individual chains tend to lie parallel to the surface while in the shifted cases they lie perpendicular to it. It should be noted that the chains in our model are not allowed to form chemical bonds with the surface.

\section{B. Choice of physical units}

The physical units are determined by the Hamaker constant $A_{H}$ of the substrate and by the number density of substrate atoms. In our units, we have fixed $\rho_{s}=1.0 \AA^{-3}$. The Hamaker constant $A_{H}$ of the substrate fixes the effective bond length $b_{l}$. This can be seen as follows: the Hamaker constant between two materials is defined by

$$
A_{H}=4 \pi^{2} \epsilon_{s} \sigma_{s}^{6} \rho_{s} \rho_{f},
$$

where $\rho_{f}$ denotes the number density of molecules in the fluid [21]. We have fixed $A_{H}$ through the choice $\epsilon_{s}=$ $2.8 \times 10^{-20} \mathrm{~J}$ and $\sigma_{s}=1.25 \times 10^{-9} \mathrm{~m}$ and requiring that 
it is realistic, i.e. $A_{H} \sim 10^{-18} \mathrm{~J}$ 21]. This fixes the number density of the fluid to be $\rho_{f} \sim 10^{24} \mathrm{~m}^{-3}$. On the other hand, $\rho_{f} \sim b_{l}^{-3}$ and hence the effective bond length $b_{l} \sim 10^{-8} \mathrm{~m}=100 \AA$. This justifies the use of a flat, continuum substrate in our model studies.

For an LJ system there is a typical time scale, which is fixed by the choice of $\epsilon_{f}, \sigma_{f}$, and the mass $m$. In our bare units, the mass of a monomer is $m_{b}=63.5$ amu, $\epsilon_{f}=0.1703 \mathrm{eV}$ and $\sigma_{f}=2.3 \AA$. The combination that yields a quantity with the dimension of time is $\tau_{c}=$ $\sqrt{\left(m_{b} \sigma_{f}^{2}\right) / \epsilon_{f}}=\sqrt{m_{b} / \epsilon_{f}} \sigma_{f}$ which in our bare units is $\approx$ $5 \times 10^{-13} \mathrm{~s}$. In our simulation algorithm, we have chosen the time step to be $0.01 \tau_{c}$. To obtain physical units we use the bond length $b_{l} \approx 100 \AA$ which scales $\sigma_{f}$, and set the physical mass of our effective monomers to be a realistic value of $m=10^{5} \mathrm{amu}$. Using these values to scale $\tau_{c}$ gives the time step in physical units to be $t_{r . u .}=7.7 \times 10^{-13} \mathrm{~s}$.

\section{Choice of thermostat}

The dynamics of the system with a Nosé-Hoover (NH) thermostat is described by the usual equations of motion [16]:

$$
\begin{gathered}
\frac{d \vec{r}_{i}}{d t}=\frac{\vec{p}_{i}}{m_{i}} \\
\frac{d \vec{p}_{i}}{d t}=-\nabla_{i} V-\eta \vec{p}_{i}
\end{gathered}
$$

and

$$
\frac{d \eta}{d t}=\left[\sum_{i} \frac{p_{i}^{2}}{m_{i}}-N_{f} k T_{s}\right] / N_{f} k T_{s} \tau^{2}
$$

where $N_{f}$ is the number of degrees freedom, $k T_{s}$ the temperature for the thermostat, $\eta(t)$ a time-dependent friction coefficient, and $\tau=2.0 \times 10^{-14} \mathrm{~s}$ is a relaxation time. If we were to study the equilibrium properties of our model, we note that this choice of $\tau$ would remove any uncanonical temperature fluctuations due to a hidden Toda demon [23]. The equations of motion are solved using modified velocity Verlet algorithm (see e.g. Ref. 24]). The simulations are performed at temperature $k T=0.8 \epsilon_{f}$ which is well above the triple point of an LJ fluid [16]. At the end of this work, we will also briefly discuss the influence of choosing a local thermostat based on Langevin dynamics to our results.

\section{Construction of the initial configuration}

We use the cylindrical geometry of Refs. 114,16, and construct an initial ridge-shaped droplet with periodic boundary conditions along the direction of the ridge which is denoted by $y$. The length of the cylinder is $\approx 10 b_{l}$ for dimers, $\approx 16 b_{l}$ for tetramers and $\approx 38 b_{l}$ for octamers. Spreading takes place in the $x$ direction which lies perpendicular to the ridge. This choice of geometry is for computational convenience, and translating our results to the true $3 \mathrm{D}$ case is straightforward.

Constructing a proper initial configuration is complicated by long relaxation times of the chain-like molecules. To overcome this, in the beginning the locations of the end-groups of the $n$-mers are chosen randomly. Then the chains are formed in such a way, that they are bent 90 degrees at each joint, while their directions are random. The initially rather sparse system is compressed to find the minimum of the internal energy. Then the droplet is allowed to equilibrate in the following way: the temperature of the system is set to $0.1 k T_{s}$, where $k T_{s}=0.8 \epsilon_{f}$ denotes the actual simulation temperature. Then the temperature is raised to $1.5 k T_{s}$ by continuously adjusting the temperature of the thermostat. All this time the system is allowed to evolve without the surface interaction. After this the temperature is lowered to $k T_{s}$ in the same way; this should result in a configuration that is closer to the actual equilibrium configuration than the initial with. We have qualitatively confirmed this by estimating the corresponding free energy differences [24]. The time scales used in constructing the initial configuration are comparable to the actual spreading simulation. After the system has been equilibrated, the substrate potential is "switched on" and spreading can take place.

\section{E. Quantities calculated}

One of the main advantages of the MD method is that the spreading dynamics can be followed in real time, and detailed data on the configurations are available. To this end, we have calculated the following quantities:

(i) The width of the precursor film $w(t)$ is a measure of the average horizontal extent of the droplet in the $x$ direction. In our geometry the spreading takes place in the $x$ direction only and hence simple geometrical arguments give $w(t) \approx N_{p}(t) /(\rho L)$, where $N_{p}(t)$ is the number of particles in the precursor film, $\rho$ denotes the average number of particles per unit area in it and $L$ denotes the length of the droplet along the $y$ axis. To convert this to the spherically symmetric 3D case we assume that the flux of particles to the precursor film $\propto t^{\alpha}$ where $\alpha \approx 1$. This immediately implies, that $w(t) \approx A t^{\alpha}$ for a compact layer. It follows then that for our geometry

$$
N_{p}(t) \approx A \rho L t^{\alpha}
$$

On the other hand, the radius of the precursor film of a fully spherical droplet that spreads radially, is given by 


$$
r(t) \approx \sqrt{N_{p}(t) /(\rho \pi)} .
$$

Combining Eqns. (8) and (9) we obtain

$$
\begin{array}{r}
r(t) \approx \sqrt{A \rho L t^{\alpha} /(\rho \pi)} \\
=\sqrt{A L / \pi} t^{\alpha / 2} \\
\equiv \sqrt{D_{e}} t^{\alpha / 2},
\end{array}
$$

where the associated early-time "diffusion" (transport) coefficient is defined by $D_{e}=L A / \pi$.

(ii) The density profile is of particular interest because it can be directly measured in the experiments. In our model it is defined as the average number of particles in a box of size $\Delta x \Delta y$ taken at any fixed $y$.

(iii) The pair correlation function characterizes the degree of order in the system. It is defined as the following configuration average [24]:

$$
g(r, t)=(N / V)^{-2}\left\langle\sum_{i} \sum_{j \neq i} \delta\left({\overrightarrow{r_{i}}}^{c m}\right) \delta\left({\overrightarrow{r_{j}}}^{c m}-\vec{r}\right)\right\rangle,
$$

where $N$ denotes the number of molecules in the system of volume $V$ and $\vec{r}_{i}{ }^{c m}$ is the position of the centre-of-mass of the $i^{\text {th }}$ molecule. In particular, we have calculated $g(r, t)$ within the effectively $2 \mathrm{D}$ precursor film, where $N \equiv N_{p}(t)$ and $V \equiv A_{p}(t)$, the latter denoting the time-dependent area of the precursor film. It should be noted that since the system is not translationally invariant in the $x$ direction, $g(r)$ starts deviating from its asymptotic values for large $r$, for which $g(r) \rightarrow 1$ when $r \rightarrow \infty$.

(iv) The distribution of orientations for neighbouring chains $d_{o}$ is defined as follows: to every chain $i$ a vector $\vec{R}_{i}$ is assigned as $\vec{R}_{i}=\vec{r}_{i+n l}-\vec{r}_{i}$. Here $\vec{r}_{i}$ denotes the position of the grafted end of the chain $i$, and $\vec{r}_{i+n l}$ denotes the position of the other end of the chain. The normalized distribution is defined as follows:

$$
d_{o}(\theta, t)=\frac{N_{o}(\theta, t)}{\sum_{\theta=0^{\circ}}^{180^{\circ}} N_{o}(\theta, t)},
$$

where $N_{o}(\theta, t)$ denotes the number of pairs of chains oriented at an angle $\theta$ relative to each other at time $t$. Only those pairs of chains whose centres of masses lie within a sphere of radius $2.5 \sigma_{f}$ are considered. This quantity indicates the spatial orientational correlations of neighbouring chains. This method is accurate when we use rigid chains, but in the case of flexible ones it gives only an approximate picture of the degree of orientational correlations between neighbouring chains. (v) Distribution of bond angles $d_{b}$ is a timedependent quantity that keeps track of bond angles at given times. The bond angle is defined to be the angle between consecutive bonds in a molecule and it is calculated by taking the dot product of the associated bond vectors. Bond vector is defined as the vector between consecutive monomers in the chain. Distribution of bond angles $d_{b}$ is defined as the following normalized histogram:

$$
d_{b}(\theta, t)=\frac{N_{b}(\theta, t)}{\sum_{\theta=0^{\circ}}^{180^{\circ}} N_{b}(\theta, t)},
$$

where $N_{b}(\theta, t)$ denotes the number of bond angles with angle $\theta$ at time $t$. It is calculated for the initial configuration and for a configuration taken at late stages of spreading. It is used in analyzing whether completely flexible chains become effectively stiffer in the course of spreading or not. This tendency would be revealed by a distribution profile peaked sharply around $\theta \approx 0^{\circ}$. This quantity is calculated for tetramers and octamers only.

\section{RESULTS}

First we consider short, rigid molecules with two monomer units. These are called dimers, and we will present results for two different sets of surface interaction parameters. Then we consider longer flexible chains, and we present results for chains with four monomer units (tetramers) and with eight units (octamers).

\section{A. Dimers}

In this section we present complete results for short and rigid molecules (see Ref. [22] for a brief summary). We have studied two different cases, namely one in which the equilibrium orientation of an individual chain is parallel to the surface (the ordinary case) and the other in which the molecules prefer to lie perpendicular to the surface (the shifted case). The shifted case can be considered to be an effective model for rigid molecules with one end hydrophobic and the other hydrophilic relative to the surface [6]. The grafted end interacts with the surface with potential $V_{\mathrm{I}}$ while in the ordinary case the other end has $V_{\text {II }}$ and in the shifted case $V_{\text {III }}$.

\section{The ordinary case}

Figs. 2(a) and (b) show a sequence of snapshots from a typical evolution of the droplet for $N=1525$ ordinary dimers during spreading as seen along the axis of the ridge. The holes in the initial configuration are due to density fluctuations. The initial configuration is characterised by a disordered and liquid-like structure. This 
is revealed by the pair correlation function and the distribution of orientations for neighboring dimers for this geometry.

After switching on the surface attraction it can be seen that the dimers in the middle of the droplet that have not yet come into contact with the surface are mainly oriented perpendicular to the surface with the grafted end pointing downwards due to stronger surface attraction. The molecules that are on the surface, on the other hand, behave quite differently. It can be seen that the precursor film is very disordered except very close to the edges of the droplet. This is due to the high density of dimers near the center of the droplet which effectively prevents them from attaining their equilibrium orientations relative to the surface. At the edges of the droplet the dimers have enough room to lie flat on the surface. The final configurational stage is a thinning monolayer of molecules lying flat on the surface and exhibiting diffusive motion.

To quantify these observations we have calculated the pair correlation function of the center of mass of the molecules within the precursor film. This case shown in Fig. 3 reveals that there is only weak short-range order characteristic of liquids. In order to characterize the degree of long-range orientational correlations we have calculated the distribution of orientations for neighbouring dimers at a late stage of spreading. It reveals that the overall orientational correlation between well-separated dimers is weak. This again is consistent with conclusions made above about the structural order within the droplet.

Fig. 4 shows the evolution of the density profile of the droplet taken at three different time steps. It can be seen that the profiles are fairly smooth and rounded; the peaks are due to dimers that have not yet come into contact with the surface. At later times a step develops at the edge of the film where dimers tend to lie flat on the surface.

Fig. 5 shows the dependence of the horizontal width of the precursor film $w(t)$ on time for a typical simulation run for $N=1525$. Qualitatively, the data look similar to that of Refs. 14, 16] with two regimes visible. We have analyzed the data as follows. First we assume that

$$
w(t)= \begin{cases}A\left(t-t_{1}\right)^{\alpha}+w_{0}, & \text { for } t_{1}<t<t_{2} \\ B\left(t-t_{2}\right)^{\beta}+w_{0}^{\prime}, & \text { for } t>t_{2}\end{cases}
$$

in which $t_{1}$ and $t_{2}$ denote cross-over times, $w_{0}$ and $w_{0}^{\prime}$ are constants, and $\alpha$ is the exponent for the first and $\beta$ for the second regime. ¿From the data one expects to find two different power law regimes. A standard trick is to estimate the initial transient time $t_{1}$ and then plot $\ln \left(w(t)-w_{0}\right)$ vs. $\ln t$. When trying to obtain $\beta$ in a similar manner, the difficulty lies in the fact that the estimation of $t_{2}$ which denotes crossover towards final $2 \mathrm{D}$ diffusion, is not as straightforward as for $t_{1}$. A linear least-squares fit for the first regime gives $w(t) \sim t^{0.8 \pm 0.1}$. The same procedure is then applied to the other regime.
The slope of the least-squares linear fit for the second "diffusive" regime gives $w(t) \sim t^{0.5 \pm 0.1}$.

These two exponents obtained from the data are consistent with the results of Refs. 14, 16, They found a crossover for the width of the precursor film $w(t)$ from "almost linear" $\left(\sim t^{0.9}\right)$ to "diffusive" $\left(\sim t^{0.5}\right)$ behavior. When we translate this to the $3 \mathrm{D}$ situation, we recover the two "diffusive" regions with different effective transport coefficients in accord with experiments [1] 3, 15. Using our result $\alpha \approx 0.8$ and extracting $A$ we find that the early-time diffusion coefficient $D_{e} \approx 5.4 \times 10^{-6} \mathrm{~m}^{2} / \mathrm{s}$. For the late time "diffusion" coefficient we find $D_{\ell} \approx$ $1.2 \times 10^{-6} \mathrm{~m}^{2} / \mathrm{s}$ and thus $D_{e} / D_{\ell} \approx 5$. These values are somewhat larger than the measured ones that range between $0.4-2.0 \times 10^{-12} \mathrm{~m}^{2} / \mathrm{s}$ [5]. Also, typical experimental ratios are of the order of $100-1000$. The difference is not surprising since in our units $T \approx 1600 \mathrm{~K}$, and there are no surface diffusion barriers. Corrugation of the surface would most likely tend to lower the late time "diffusion" coefficient thereby making the ratio larger. We note that extrapolating our values of $D$ to room temperatures gives about $10^{-15} \mathrm{~m}^{2} / \mathrm{s}$, in reasonable agreement with experiments.

The fact that $w$ is a function of both time $t$ and the number of dimers $N$ suggests that there might exist a scaling form for $w(t, N)$ as suggested in Ref. [16, which is of the following form:

$$
w(t)=t^{x} \Phi\left(t / N^{y}\right) .
$$

However, for the present case we find no such scaling for the range of times and system sizes studied. This is in part because for our relatively small systems, crossover to diffusive behavior is very sharp and thus the data do not collapse.

\section{Shifted case}

For the shifted case, Figs. 6(a) and (b) show a typical evolution of the droplet for $N=1525$ shifted dimers during spreading. Initial configuration is identical to that of the ordinary case. Again, the dimers that have not yet come into contact with the surface behave in a manner similar to the ordinary case. The molecules in the middle of the droplet are mainly oriented perpendicular to the surface. A striking difference between the ordinary and the shifted case is the development of a compact precursor layer which appears very well ordered at all stages of spreading. Fig. 7 shows the pair correlation function within the precursor film for the shifted case taken at $t=80000$ r.u.. Clear peaks can be observed corresponding up to about fourth or fifth nearest neighbor dimers. The precursor layer in this case indicates a high degree of local ordering even at these elevated temperatures.

We have also calculated the distribution of orientations for neighbouring dimers for the shifted case. The overall shape of the profile, which is shown in Fig. 8 corresponding to late stages of spreading, reflects the proper- 
ties discussed above. A clear peak can be seen indicating orientation of nearby dimers in a preferred (vertical) direction. In this case the orientational correlation of the dimers extends through the entire precursor layer.

Fig. 9(a) shows typical density profiles of dimers taken at three different times. Whereas in the ordinary case a step developed at the edges of the droplet, in the shifted case the edge of the precursor film always remains very sharp and well-defined. It is interesting to compare the calculated density profiles with the experimental ones shown in Fig. 9(b) for triloxane polyoxyethylene molecules spreading on silica bearing a dense grafted layer of trimethyls [6]. In this case grafting results in a hydrophobic surface. The triloxane polyoxyethylene is a hammer-shaped molecule which has a hydrophobic (the trisiloxane head) and a hydrophilic (polyoxyethylene tail) part. The attraction of the hydrophobic group to the surface and the repulsion between the hydrophilic part and the surface forces the molecules lie perpendicular to the surface. Despite the enormous difference in the horizontal scales, the simulated and experimental profiles are in good qualitative agreement.

Fig. 10 shows the dependence of $w(t)$ for the shifted case. Again a crossover form "almost linear" to "diffusive" behavior can be seen. The crossover appears to be somewhat sharper in this case. With the smallest system size studied the spreading stops completely due to finite-size effects. With larger systems the spreading continues in a diffusive manner. We have analyzed the data for $w(t)$ in the same way as before, and find that within the "almost linear" regime $w(t) \sim t^{0.8 \pm 0.1}$ and in the "diffusive" regime $w(t) \sim t^{0.4 \pm 0.1}$. We again convert these results into the $3 \mathrm{D}$ case and recover the two diffusive regions. We estimate that $D_{e} \approx 5.4 \times 10^{-6} \mathrm{~m}^{2} / \mathrm{s}$, and $D_{\ell} \approx 1.1 \times 10^{-7} \mathrm{~m}^{2} / \mathrm{s}$ which give $D_{e} / D_{\ell} \approx 50$, in good agreement with experiments [5].

The reason for $D_{\ell}$ being about an order of magnitude smaller than in the ordinary case can be understood from simple energetic arguments. The activation energy for diffusion in the shifted case is larger due to the high degree or local ordering. We have estimated the activation energies $E_{a}$ for diffusion for the two cases by calculating the average energy due to neighbours of a dimer located within two bond lengths from the edges of the droplet. In the ordinary case we find $E_{a} \approx 0.9 \mathrm{eV}$ and in the shifted case $E_{a}^{\prime} \approx 1.2 \mathrm{eV}$. If we make the assumption that $D_{\ell}$ follows the Arrhenius form $D_{\ell} \propto e^{-E_{a} / k T}$, we can estimate that

$$
\frac{e^{-E_{a} / k T}}{e^{-E_{a}^{\prime} / k T}} \approx 10
$$

This is fully consistent with results extracted from the width of the precursor film.

In the case of shifted dimers we again checked the scaling form of Eq. (16), but did not find a good data collapse for the present times and system sizes studied.

\section{B. Tetramers}

For chains consisting of four monomers we have studied three different cases, namely two ordinary cases (rather stiff and completely flexible tetramers) and a completely flexible shifted case. The surface potential for the grafted end is set to be $V_{\mathrm{I}}$ and for the ordinary cases we employ the surface potential $V_{\mathrm{IV}}$. In the shifted case the surface potential of the other end is set to $V_{\mathrm{V}}$, whereas the monomers in the middle of the chain do not have any interactions with the surface.

\section{Rather stiff tetramers}

Rather stiff tetramers are characterised by a fairly strong angle-dependent potential between consecutive bonds in a chain, namely $V_{\theta}=\epsilon_{\theta}(\cos \theta+1)$, where $\epsilon_{\theta}=10 \epsilon_{f}$. Figs. 11(a) and (b) show the evolution of a droplet for $N=785$ in a typical simulation run. The chains appear to be initially fairly straight and the calculation of the distribution bond angles confirms this observation. The apparent holes in the droplet are due to thermal fluctuations. The calculation of distribution of orientations for neighbouring chains also supports the conclusion that the structure of the initial configuration is disordered and liquid-like.

Qualitatively, the results are similar to the case of ordinary dimers. The chains in the middle of the droplet are oriented approximately perpendicular to it, the grafted end being closest to the surface. Closer to the edges, the chains tend to attain a more horizontal orientation. The centre of the droplet appears to have a very complicated structure. This is due to the finite flexibility of chains, as calculation of the bond angle distribution reveals that the chains are bent slightly more than in the initial configuration.

We have also calculated the pair correlation function within the precursor layer corresponding to late stages of spreading. The structure of the precursor film in this case is disordered and liquid-like. At very late stages the neighboring chains tend to orient themselves in the same direction. This is revealed by calculating the distribution of orientations. The final stages of spreading correspond to diffusively thinning monolayer where the chains prefer to be relatively straight.

The density profiles of Fig. 12 are characterised by a rounded overall shape with a peak corresponding to the chains that are not yet in the precursor film. This is consistent with the observations made from the configurations of Fig. 11. Fig. 13 shows $w(t)$ for three different system sizes. A noteworthy feature is that the crossover towards late-time diffusive behavior is not as clear as in the case of dimers. For the largest system size we find that $w(t) \sim t^{0.8 \pm 0.1}$ within the initial "almost linear" regime, but our data for this system size does not extend far enough to capture the second "diffusive" regime. For the smallest system size $N=505$ we find that in the "al- 
most linear" regime $w(t) \sim t^{0.8 \pm 0.1}$ and in the "diffusive" regime $w(t) \sim t^{0.5 \pm 0.1}$. ¿From these data we estimate that $D_{e} \approx 5.0 \times 10^{-6} \mathrm{~m}^{2} / \mathrm{s}$ and $D_{\ell} \approx 7.0 \times 10^{-7} \mathrm{~m}^{2} / \mathrm{s}$ which give $D_{e} / D_{\ell} \approx 7$.

For the present case, we also find a good data collapse using the scaling form of Eq. (16), with $x=0.9$ and $y=0.9$. Fig. 14 shows the scaling function $\Phi(z) \sim$ const. for $z \ll 1$, and $\Phi(z) \sim z^{1 / 2-x}$ for $z \gg 1$ [16.

\section{Completely flexible tetramers}

The results for completely flexible tetramers are very similar to the results for rather stiff ones. However, in this case the density profiles in Fig. 15 appear to be somewhat flattened at late stages as compared to the rather stiff case. This is evidently due to the greater flexibility of the chains. The width of the precursor film for $N=505$ tetramers gives $w(t) \sim t^{0.9 \pm 0.1}$ in the "almost linear" regime, and $w(t) \sim t^{0.5 \pm 0.1}$ in the "diffusive" regime. We find $D_{e} \approx 4.5 \times 10^{-6} \mathrm{~m}^{2} / \mathrm{s}$ and $D_{\ell} \approx 2.6 \times 10^{-7} \mathrm{~m}^{2} / \mathrm{s}$ which give $D_{e} / D_{\ell} \approx 20$. Scaling of Eq. (16) is again obeyed, with $x=0.9$ and $y=0.9$.

\section{Completely flexible tetramers - shifted case}

For the shifted tetramer case, the grafted end has the usual surface potential $V_{\mathrm{I}}$ whereas the other end had potential $V_{\mathrm{V}}$ for which the equilibrium distance from the surface extends about three bond lengths further away. We thus expect the results to be similar to those for the shifted dimers, with possible differences arising from the greater configurational entropy of the chainlike molecules. Here we mostly concentrate in the morphology of the droplets during spreading.

Figs. 16(a) and (b) show the evolution of the droplet for $N=1010$. It can be seen that the evolution is strikingly different from the ordinary case. As in the case of shifted dimers, the precursor films appears to be very compact and well-ordered. We have calculated the pair correlation function within the precursor film, which is shown in Fig. 17. Clear peaks corresponding up to about fourth or fifth nearest neighbour chains are clearly present, which is an indication of a high degree of local ordering. If one compares the pair correlation functions between shifted dimers and tetramers, it can be seen that for tetramers the peaks appear to be somewhat broadened. This is evidently due to the chain-like structure of the tetramers. Thus, with the present choice of interactions the influence of chain flexibility is rather small even at high temperatures.

We have also followed the time evolution of the density profile of the droplet. This is shown in Fig. 18. The profile develops from a fairly sharply peaked one towards a smooth but compact form. These profiles again confirm the observations made from the snapshots. We have estimated the effective diffusion barriers at late times and find that $E_{a}^{\prime} \approx 2.9 \mathrm{eV}$ compared to $\approx 1.2 \mathrm{eV}$ for the shifted dimer case. This can be understood on the basis that within a well--ordered layer, the number of neighbors for tetramers should be roughly more than twice the corresponding number for dimers.

\section{Octamers}

The results for chains built up of eight monomers (octamers) are presented in this section. We have studied two different systems, namely one in which the chains are rather stiff and another in which the chains are completely flexible. The grafted end has the usual surface potential $V_{\mathrm{I}}$ whereas the other monomers have $V_{\mathrm{IV}}$. It should be pointed out that due to CPU-time constraints out system sizes are relatively small.

\section{Rather stiff octamers}

Initial configuration for a droplet of rather stiff octamers $\left(\epsilon_{\theta}=10 \epsilon_{f}\right)$ of size $N=488$ is shown in Fig. 19(a). It is characterised by a fairly complex structure. The tendency of the chains to be relatively straight is clearly visible. Fig. 19(b) shows a typical evolution of the droplet. The structure in the middle of the precursor film is very complex. Chains at the edges of the droplet again tend to lie parallel to the surface. We have calculated different time--dependent quantities such as the pair correlation function within the precursor film taken at late stages of spreading. From the shape of the function we can immediately conclude that the precursor film is disordered and liquid-like. The directions of neighbouring chains are fairly strongly correlated, however. Calculation of the distribution of bond angles reveals that the chains tend to remain fairly straight through the whole spreading process.

A set of typical density profiles are shown in Fig. 20. It can be seen that at later times they become rather smooth and rounded. For the width of the precursor film we find that initially $w(t) \sim t^{0.9 \pm 0.1}$ with $D_{e} \approx 1.7 \times 10^{-5} \mathrm{~m}^{2} / \mathrm{s}$. For the late-time behavior, we find that $w(t) \sim t^{0.5 \pm 0.1}$ and $D_{\ell} \approx 4.3 \times 10^{-6} \mathrm{~m}^{2} / \mathrm{s}$. For the ratio we thus find $D_{e} / D_{\ell} \approx 4$. As far as the scaling is concerned, we were not able to collapse the data for different system sizes for the present case.

\section{Completely flexible octamers}

The results for $N=488$ completely flexible octamers are very similar to results for the rather stiff ones. Again the precursor film is disordered and a crossover from "almost linear" $\left(w(t) \sim t^{0.8 \pm 0.1}\right)$ towards "diffusive" $\left(w(t) \sim t^{0.5 \pm 0.1}\right)$ behavior for $w(t)$ is recovered, with $D_{e} \approx 3.7 \times 10^{-5} \mathrm{~m}^{2} / \mathrm{s}$ and $D_{\ell} \approx 1.77 \times 10^{-6} \mathrm{~m}^{2} / \mathrm{s}$, which yield $D_{e} / D_{\ell} \approx 20$. The density profiles shown in Fig. 21 bear a close resemblance to the ones for rather 
stiff octamers, the late-time profiles being somewhat less rounded in this case.

\section{The influence of Langevin dynamics}

We have also implemented Brownian dynamics into our computer code, the motivation being to study the influence of a local thermostat for the present case. Recently, the NH thermostat has been claimed to be physically unsuitable for microscopic studies of droplet spreading [19], despite the fact that for a small system out of equilibrium, there is no unique "best" choice. We note that since we have a smooth surface, coupling to an auxiliary thermostat must be used here.

To check our results, we have performed additional simulations for $N=555$ shifted dimers [7]. We have employed two different values for the friction coefficient $\eta$, namely $\eta_{1}=3 \times 10^{14} \mathrm{~s}^{-1}$ and $\eta_{2}=0.3 \times 10^{14} \mathrm{~s}^{-1}$. We have set our bare time step to $0.01 \times 10^{-14} \mathrm{~s}$. For $\eta_{1}$ we recover the two different regimes for $w(t)$ with $w(t) \sim t^{1.0 \pm 0.1}$ and $w(t) \sim t^{0.5 \pm 0.1}$. We can again extract the associated "diffusion" coefficients with the result that $D_{e} \approx 5 \times 10^{-6} \mathrm{~m}^{2} / \mathrm{s}$ and $D_{\ell} \approx 1.5 \times 10^{-7} \mathrm{~m}^{2} / \mathrm{s}$ for the early and late time regime, respectively. For the ratio we thus find $D_{e} / D_{\ell} \approx 30$. Calculation of the pair correlation function within the precursor film again reveals that the layer has a high degree of local order.

For $\eta_{2}$ we also recover the two regimes with $w(t) \sim$ $t^{0.9 \pm 0.1}$ and $w(t) \sim t^{0.4 \pm 0.1}$. For this particular case $D_{e} \approx 8 \times 10^{-5} \mathrm{~m}^{2} / \mathrm{s}$ and $D_{\ell} \approx 4.0 \times 10^{-7} \mathrm{~m}^{2} / \mathrm{s}$ for the early and late time regimes, respectively. For the ratio we obtain $D_{e} / D_{\ell} \approx 200$. Reducing the friction would further increase this ratio since the late-time regime is dominated by effective diffusion barriers that are independent of $\eta$.

Based on our additional results we can conclude that qualitatively and quantitatively our results and conclusions are unaffected by the choice of the thermostat. The main effect of the local thermostat with respect to the NS thermostat is a slightly smoother crossover towards the late-time regime.

\section{CONCLUSIONS AND DISCUSSION}

In this work we have studied a simple model of dynamics of spreading for rigid and flexible molecules that interact asymmetrically with respect to a solid surface. We have studied two different cases. In the ordinary case, the end potentials are of different strength, but the equilibrium position of the molecules on the surface is horizontal. In the shifted case, however, the other end of the molecule has an equilibrium distance that is compatible with the length of the chain, i.e. the equilibrium position is vertical with respect to the surface. The latter case in particular can be considered as an effective model for the case of hydrophobic and hydrophilic surface interactions [6].

One of our main results is that the microscopic structure in the precursor film drastically depends on the nature of the asymmetrical interactions. For the shifted case, there is a high degree of local order present which makes the density profile of the droplet unusually sharp and flat. This result is in good qualitative agreement with a recent experiment on a physically similar system [6]. Moreover, our model recovers the overall $t^{0.5}$ time dependence of the radius of the precursor film, and we have been able to quantitatively estimate the associated transport coefficients. Typical ratios of the earlytime coefficients $D_{e}$ to the late-time ones $D_{\ell}$ are in very good agreement with the experiments. Furthermore, our model demonstrates how this ratio increases with increasing local order in the precursor film, in cases where the late-time diffusion is controlled by energy barriers arising from neighboring molecules.

Recently, the choice of the global NH thermostat used here and in Refs. 11, 16, has been criticized by De Coninck et al. 19] on basis of the argument that in an inhomogeneous system a global thermostat is not physically justified. However, due to our smooth surface the heat must dissipated by other means than coupling to substrate atoms held at constant temperature, as was done in Ref. [19]. Moreover, it is a well known fact that there is no unique way of controlling the instantaneous temperature of a non-equilibrium system. We have performed our simulations mainly with the NH thermostat, but test runs with Brownian dynamics reveal that the same qualitative and quantitative behavior persists. Moreover, the results of Ref. [16] as well as those of the present work compare very well with the simulations of De Coninck et al. 19], once differences in the geometries (cylindrical vs. spherical) are properly accounted for. De Coninck et $a l$. found that for largest droplets the number of atoms in the first (i.e. the precursor) layer was well described by $N(t) \sim t^{0.85 \pm 0.05}$ and the corresponding radius by $R^{2}(t) \sim t^{0.82 \pm 0.06} \sqrt{19}$. On the other hand, in our geometry $w(t) \sim N(t) \sim t^{0.9 \pm 0.1}$ for the "almost linear" regime. The flux of particles into the precursor layer is the same in the two independent studies and therefore the results are equivalent. The fact that the slower latetime "diffusive" regime was not reported in Ref. [19] is probably due to insufficient simulation times, since their system sizes were rather large. Thus both the qualitative and quantitative features of the spreading phenomenon are fairly insensitive to the choice of thermostat as well as the geometry.

To summarize, we hope to have further demonstrated in this work that the spreading phenomenon at microscopic length-scales is a very complicated process. It seems highly unlikely that the properties of all the different cases studied here and in other works could be obtained from a more general framework. On the other hand, there are many features of spreading, such as the time--dependence of the precursor radius that are rather 
insensitive to the details of interactions, or molecular structure of the liquid. This work calls for more systematic and controlled experimental as well as theoretical work in order to further classify the properties of tiny liquid droplets spreading on a solid surface.

[1] F. Heslot, N. Fraysse, and A. M. Cazabat, Nature 338, 640 (1989).

[2] F. Heslot, A. M. Cazabat, and P. Levinson, Phys. Rev. Lett. 62, 1286 (1989).

[3] F. Heslot, A. M. Cazabat, P. Levinson, and N. Fraysse, Phys. Rev. Lett. 65, 599 (1990).

[4] U. Albrecht, A. Otto, and P. Leiderer, Phys. Rev. Lett. 68, 3192 (1992).

[5] M. P. Valignat, N. Fraysse, A. M. Cazabat, and F. Heslot, Langmuir 9, 601 (1993).

[6] A. M. Cazabat, N. Fraysse, F. Heslot, P. Levinson, J. Marsh, F. Tiberg, and M. P. Valignat, Adv. Coll. Int. Sci 48, 1 (1994).

[7] M. Haataja, J. A. Nieminen, and T. Ala-Nissila, unpublished (1995).

[8] T. Ala-Nissila, S. Herminghaus, T. Hjelt, and P. Leiderer, unpublished (1996).

[9] D. B. Abraham, P. Collet, J. De Coninck, and F. Dunlop, Phys. Rev. Lett. 65, 195 (1990).

[10] P.-G. de Gennes and A.-M. Cazabat, C. R. Acad. Sci. Paris Ser. II 310, 1601 (1990).

[11] This holds, provided that the ratio of the radius of the precursor film and the radius of the second layer remains approximately constant.

[12] J. Heiniö, K. Kaski, and D. B. Abraham, Physica Scr. T38, 28 (1991).

[13] J.-x. Yang, J. Koplik, and J. R. Banavar, Phys. Rev. Lett. 67, 3539 (1991); Phys. Rev. A 46, 7738 (1992).

[14] J. A. Nieminen, D. B. Abraham, M. Karttunen, and K. Kaski, Phys. Rev. Lett. 69, 124 (1992).

[15] J. De Coninck, N. Fraysse, M. P. Valignat, and A. M. Cazabat, Langmuir 9, 1906 (1993).

[16] J. A. Nieminen and T. Ala-Nissila, Europhys. Lett. 25, 593 (1994); Phys. Rev. E 49, 4228 (1994).

[17] O. Venäläinen, T. Ala-Nissila, and K. Kaski, Europhys. Lett. 25, 125 (1994); Physica A 210, 362 (1994).

[18] L. Wagner, Phys. Rev. E 51, 499 (1995).

[19] J. De Coninck, U. D'Ortona, J. Koplik, and J. R. Banavar, Phys. Rev. Lett. 74, 928 (1995).

[20] S. T. Milner, Science 251, 905 (1991).

[21] J. Israelachvili, Intermolecular \& Surface Forces, 2nd ed., Academic Press, London, U.K. (1992).

[22] M. Haataja, J. A. Nieminen, and T. Ala-Nissila, Phys. Rev. E 52, R2165 (1995).

[23] B. L. Holian, A. F. Voter, and R. Ravelo, Phys. Rev. E 52, 2338 (1995).

[24] M. P. Allen and D. J. Tildesley, Computer Simulation of Liquids, Oxford University Press, Oxford, U.K. (1987). 


\begin{tabular}{|c|c|c|}
\hline \hline \multicolumn{3}{|c|}{ Surface interaction parameters } \\
\hline$\epsilon_{i}$ & $\sigma_{i}$ & Symbol \\
\hline $1.0 \epsilon_{f}$ & $5.0 \sigma_{f}$ & $V_{\mathrm{I}}$ \\
\hline $0.06 \epsilon_{f}$ & $5.0 \sigma_{f}$ & $V_{\mathrm{II}}$ \\
\hline $0.02 \epsilon_{f}$ & $7.3 \sigma_{f}$ & $V_{\mathrm{III}}$ \\
\hline $0.006 \epsilon_{f}$ & $5.0 \sigma_{f}$ & $V_{\mathrm{IV}}$ \\
\hline $0.01 \epsilon_{f}$ & $8.0 \sigma_{f}$ & $V_{\mathrm{V}}$ \\
\hline \hline
\end{tabular}

TABLE I. Surface interaction parameters and their symbols used in this study. 


\section{FIGURE CAPTIONS}

Fig. 1. Schematic illustration of the different surface potentials used in this study. The "grafted" end always has potential $V_{\mathrm{I}}$.

Fig. 2. (a) Initial configuration for the ordinary case of $N=1525$ dimers. The grafted end is represented by a large filled circle. (b) Same system taken at $t=$ 30000 r.u..

Fig. 3. Pair correlation function within the precursor film at $t=80000$ r.u.. Note the disordered and liquid-like structure of the film.

Fig. 4. Density profiles for the case of ordinary dimers. The late-time shoulders are due to the dimers that lie flat on the surface. These and all the other density profiles have been smoothed to remove noise.

Fig. 5. The width of the precursor film $w(t)$ for the ordinary dimer case. It can be characterised by an "almost linear" regime which crosses over to a "diffusive" one.

Fig. 6. (a) Initial configuration for the shifted case of $N=1525$ dimers. (b) Same system taken at $t=$ 30000 r.u..

Fig. 7. Pair correlation function within the precursor film at $t=80000$ r.u.. Clear peaks can be observed corresponding up to fourth or fifth nearest neighbour, indicating that the film displays a high degree of local order.

Fig. 8. Distribution of orientations $d_{o}$ for the shifted dimer case taken at $t=80000$ r.u.. Orientations of dimers are correlated over the whole system.

Fig. 9. (a) Density profiles for the shifted dimer case. (b) Experimental density profiles for triloxane polyoxyethylene molecules (hydrophobic head with a hydrophilic tail) spreading on silica bearing a dense grafted layer of trimethyls [6]. The profiles are strikingly similar.

Fig. 10. $w(t)$ for the shifted dimer case. It can be characterised by an "almost linear" regime which crosses over to "diffusive" one. The late-time "diffusion" coefficient is roughly one order of magnitude smaller than for the ordinary case.

Fig. 11. (a) The initial configuration for $N=785$ rather stiff tetramers. (b) Same system taken at $t=$ 80000 r.u.. Notice the complicated and disordered structure of the precursor filmi due to the flexibility of the chains.

Fig. 12. Density profiles for rather stiff tetramers taken at three different times. The profiles are fairly smooth and rounded.

Fig. 13. $w(t)$ for rather stiff tetramers with three different system sizes.

Fig. 14. Scaled data for $w(t)$ for the three different system sizes with $x=0.9$ and $y=0.9$.

Fig. 15. Density profiles for completely flexible tetramers taken at three different times. The profiles are somewhat flatter as compared to the rather stiff case due to the flexibility of the chains.

Fig. 16. (a) Initial configuration for the shifted case of $N=1010$ completely flexible tetramers. (b) Same system taken at $t=80000 \mathrm{r}$.u.. Notice the appearance of a compact precursor film with sharp edges.

Fig. 17. Pair correlation function within the precursor film at $t=80000 \mathrm{r} . u$.. Clear peaks can be observed corresponding up to fourth or fifth nearest neighbour, indicating that the film displays a high degree of local order.

Fig. 18. Density profiles for the shifted case of completely flexible tetramers taken at three different times. 
Fig. 19. (a) Initial configuration for the case of $N=$ 488 rather stiff octamers. (b) Same system taken at $t=$ 80000 r.u.. The structure of the precursor film is again disordered and liquid-like.

Fig. 20. Density profiles for the case of rather stiff octamers taken at three different times. The profiles are fairly smooth and rounded.

Fig. 21. Density profiles for the case of completely flexible octamers taken at three different times. The profiles are somewhat flatter than in the case of rather stiff octamers. 